\title{
Incongruity Theory Applied in Dynamic Adaptive Game Artificial Intelligence
}

\author{
Kevin N. Francisco, Johann-Paulo L. Ruste, Ria A. Sagum, Yun Seop Song, and Darl Sander G. Sy
}

\begin{abstract}
The Theory of Incongruity is a theory in psychology that states that people create mental concepts about the world in two forms/concepts such as the context and system complexity. The researchers presents an adaptive A.I in which it interacts with a real person and incorporates the theory in order to monitor how the user feels while playing or interacting with the A.I. Two algorithms will be utilized in order to achieve the desired adaptability of the A.I. namely, dynamic scripting and case base reasoning. The goal of the study is to create an A.I. that will be able to react fast and adapt to real human players based on how they feel or what they think regarding the decisions made by the A.I. This study aims to give a player a more thrilling and exciting experience thus, improving the quality of entertainment games provide. The study also focuses on pulling the gamer up the scale in order to improve his skills in game. Giving the player a chance to win by scaling his current skill level but still targeting in the end to win makes the adaptive A.I. more fun to interact with and prove to give a positive reaction or experience to the gamer.
\end{abstract}

Index Terms - Adaptive artificial intelligence, games, theory of incongruity, dynamic scripting.

\section{INTRODUCTION}

Video gaming is one of the most evolving types of entertainment nowadays. Providing quality entertainment and a thrilling experience to the user is the objective of any game developer. To achieve this objective and make it possible, developers since the past years have tried to make the gamer or rather the player feel like he/she is competing with a computer but with human like behavior, commonly known as Artificial Intelligence. This is the study and design of intelligent agents, where an intelligent agent is a system that perceives its environment and takes actions that maximize its chances of success automatically and adaptively Narrowed down to a type of A.I. known as Adaptive Learning, systems that endeavor to transform the learner from passive receptor of information to collaborator in the educational process, with this, the Theory of Incongruity, Dynamic Scripting, together with the key foundations of the study will be put together as one and implemented on a common gaming platforms.

Summarizing all concepts and theories used, this study is

2012; revised February 23, 2013. This work was supported in part by the University of Santo Tomas. Incongruity Theory Applied in Dynamic Adaptive Game Artificial Intelligence.

R. A. Sagum is with the faculty of Engineering University of Santo Tomas Philippines (e-mail:riasagum31@yahoo.com)

K.N. Francisco, J. L. Ruste, Y. S. Song, and D. S. Sy are with the University of Santo Tomas (e-mails: kev_may92@yahoo.com, Paulo_bluelake92@yahoo.com, yunseopsong@yahoo.com, sanderme_10@yahoo.com). all about the development and implementation of a modern concept of A.I. The study focuses on modern interactive computer game, real time strategy, where in this type of gaming concept the proposed A.I will be implemented, because it is the type of gaming experience that is popular among the "gamers". The study is important for different people whether the user is a gamer or not. Game Developers and the game companies will benefit greatly with the concept because they can apply the A.I. in their game platforms and be able to provide quality experience and thrilling sensation for their clients. Gamers and enthusiasts can utilize the application of the study for they are the ones to use and experience the intelligence of the A.I. directly thus, providing them with a very exciting and complex gaming experience without sacrificing the entertainment value of the game.

Fellow researchers and A.I. developers may benefit as well from the study by providing them with another kind of hybrid artificial intelligence that can be used as their reference in developing future versions. It is also believed that the study will increase and add information to the pool of knowledge in the field of computer science.

\section{RELATED WORK}

\section{A. Artificial Intelligence for Games}

Also known as the intelligence of machines and the branch of computer science that aims to create it, A.I. is also defined as the science and engineering of making intelligent machines. Basically, A.I. is developed to let machines mimic the intelligence of a real person in making decisions and doing actions (McCarthy, James) . Nowadays A.I. was already implemented in computer games to provide a challenging and interactive gaming experience. [1]

\section{B. Extending Dynamic Scripting}

The study focuses on extending dynamic scripting. This extended dynamic script will improve the learning performance, applicability and flexibility of the algorithm for modern games. In the research, three specific extensions were mentioned. First, is developing a flexible and stand alone, version of dynamic scripting that will allow" hierarchical dynamic scripting". Second, is to extend the context sensitivity of hierarchical dynamic scripting through automatic abstract state construction. Lastly, is to perform architecture integration where this algorithm is integrated with existing hierarchical behaviourmodelling structure. Their main objective is to help create useful frame work for game developers. The study will also help A.I. developers to easily define adaptive behaviours that utilize dynamic scripting-based learning techniques. The study also contains a lot of information about dynamic scripting and reinforced 
learning algorithms which the researchers believe is very useful for A.I development [2].

\section{Player Modeling for Intelligent Difficulty Adjustment}

The researchers aim at automatically adjusting the difficulty of computer games by clustering players into different types and supervised prediction of the type from short traces of gameplay. They induce a difficulty model and build it into the game. The actual players would not notice any of the things and they will be always challenged by the difficulty level that is estimated to be just right for them.

The researchers focus on the question of how a game can adapt to a particular player type by the data collected from the alpha/beta-testing stages and the data collected from the new player. Starting with allowing the gathering of sources by letting the computer play with different levels of people and then letting it think and apply what it has learned from the new player. Given the new player will be put to a cluster it belongs to. What they will be using was developed through XNA were a player shall control a cannon and shoots spaceships. In the end, experimental results show that dynamic adjustment and cluster prediction together outperform the alternatives significantly [3].

\section{Incongruity-Based Adaptive Game Balancing}

The research deals with a way of game balancing for entertainment value for players of different skills by using the theory of incongruity. The incongruity theory states that every context has a different level of complexity. Incongruity is defined as is the difference between the complexity of a context and the complexity of the internal human model of the context. When the incongruity is too large for the human, the human loses interest in the context decreasing the entertainment value of the game. This will conclude that boredom and frustration will cause a high positive incongruity while pleasure is a negative incongruity, so making the main goal to be a context complexity that is roughly equal to or slightly higher than system complexity.

By using a self-developed game name glove, it was derived from the classic game Gauntlet. The basic game has three difficulty levels, namely, easybalanced, and hard. When the difficulty level is easy, the game aims at having the knight win the game with about $50 \%$ of his health remaining. When the difficulty level is 'hard', the game aims at having the knight lose the game when he has progressed through about $50 \%$ of the game world. When difficulty level is 'balanced', the game aims at having the knight experience a narrow victory or a narrow defeat. The game accomplishes the result envisaged by controlling the number and types of spawned enemies. In this way the easy and hard levels try to keep the incongruity stable and high. The test was conducted on a group of subjects who will play a game with three difficulty settings. The idea was to maintain a specific difference in incongruity automatically. The main conclusion was that, due to their automatically maintained balanced difficulty setting, they were able to avoid the game from becoming boring or frustrating [4].

\section{E. Opponent Modelling for Case-based Adaptive A.I}

This research is just a continuation from an previous work of them in which they introduced a novel approach to adaptive game AI that was focused on the rapid and reliable adaptation to game circumstances namely 'case-based adaptive game AI'. In the approach, domain knowledge required to adapt to game circumstances is gathered automatically by the game AI, and is exploited to evoke effective behaviour in a controlled manner in online play. In the research they investigated to what extent incorporating opponent modeling enhances the performance of case-based adaptive game AI, and by their approach, models of the opponent players are generated automatically, on the basis of observations drawn from a multitude of games. They performed experiments that test the enhanced approach in an actual, complex RTS game, and observed that the effectiveness of case-based adaptive game AI increases significantly when opponent modeling is incorporated. [5]

\section{F. Case-Based Planning and Execution for Real-Time Strategy Games}

Due to the problems of traditional artificial intelligence techniques, as it fails to play at a human level because of the vast search spaces that they entail. The researchers presented a real-time case based propose to extract behavioural knowledge from expert demonstrations in form of individual cases. This knowledge can be reused via a case based behaviour generator that proposes behaviours to achieve the specific open goals in the current plan.

After some promising results, the main contributions of the framework are:

1) A case based integrated real-time execution and planning framework.

2) The introduction of a behaviour representation language that includes declarative knowledge as well asprocedural knowledge to allow both reasoning and execution.

3) The idea of automatic extraction of behaviors from expert traces as a way to automatically extract domain knowledge from an expert.

4) The idea of heterogeneous case bases where cases that contain solutions for several different problems and

5) The introduction of delayed adaptation to deal with dynamic environments. [6]

\section{G. A CBR Inspired Approach to Rapid and Reliable} Adaptation of Video Gaming

Being uncontrollable and unpredictable is the major problem why adaptive A.I.is hampered from being implemented on commercial games. In this study they have introduced an alternative approach to tackle this problem. Case based inspired approach is the core of this study. This approach gathers domain knowledge required to adapt is automatically gathered by the A.I. and exploited immediately to evoke effective behavior in a controlled manner in online play. They have introduce a 3 step model to implement this approach namely offline processing, initialization and online adaptation. Based from the result of their study CBR provides a strong basis for effectively adapting game A.I. in video games. [7]

\section{H. Case-Based Plan Recognition in Computer Games}

In their paper they explored the use of case-based plan recognition to predict a player's actions in a computer game by testing it in the Space Invaders game. They present a prototype plan recognition system called COMETS that uses 
the case-based plan recognition idea. The idea is to have the NPC observe the player's behavior and identify plans or patterns that recur. Then the NPC can identify future executions of these plans and anticipate what the user will do next. In the case-based plan recognition (CBPR) methodology the observed plans are stored in a case-base (plan library) and the player's behavior is constantly compared to the case-base to identify the onset of the execution of a recognized plan. [8]

\section{Dynamic Programming (Algorithms 2007)}

This is the Chapter 6 of the book discussing dynamic programming. The book emphasized the directed acyclic graph (DAG) which lies in the heart of dynamic programming. Being able to make the graph linear is the special distinguishing feature of DAG. The core discussion of this book is how dynamic programming works. It is stated in the book that first it tries to identify collections of sub problems and tackling them one by one smallest first. Then by using the answers that have been derived from the small sub problems it will now solve or figure out larger ones. As we go along the chapter of the book examples problems and diagrams was given about dynamic programming which greatly helped us to understand how it works. [9]

\section{J. Dynamic Formations in Real Time Strategy Games}

The study tackles about organizing units or agents in strategic video games. They have discussed the difference between the use of static and dynamic formations. Like the difference between a static and dynamic A.I. Unit formation also greatly affects the adaptability of the agents that is being controlled by the A.I. Their paper they discussed and approach to organizing units by learning the effectiveness of formation in actual play, and directly applying learned formation according to the classification of the opponent player. ORTS or commonly known as open real time strategy has been used as a testing ground for their approach. The yielded results showed that dynamic formation can be applied to video games.[10]

\section{K. Automatic Rule Ordering for Dynamic Scripting}

The ability to self correct mistake and creativity in responding to new situation has been the goal of adaptive A.I. This paper also utilizes dynamic scripting to be able to achieve the goal of an adaptive A.I. We know that dynamic scripting utilizes rules contained inside a rule base or knowledge base. The study tried to develop and create an improvement for dynamic scripting which will make an automatic rule ordering for the knowledge base. Three different automatic rule - ordering mechanism for dynamic scripting was implemented namely WeightOrdering, Relation - Weight Ordering and Relation Weight Ordering with Selection Bonus. The results of the study showed that using an automatic rule ordering is at least as effective as dynamic scripting with manually tuned priority values. It also has the ability to create a novel game A.I. with significantly increased effectiveness. [11]

\section{SYSTEM MODEL}

The whole system starts from the two offline processes that occur during the start-up of the game platform. Clustering of observation and game indexing are the two components of offline processing with a subcomponent namely, clustered observation and game indices. All observations and rewards that are processed and saved by the A.I. will be prepared in this part of the process. At first, the agent will start with strategies based on the current game state. Using the similarity matching capabilities of the A.I. it will now choose the most similar observation from the clustered information to the current state of the game.

The next phase will now be the adaptability part of the A.I. Online strategy selection will be the core of the A.I. Agents execute decisions and actions done during the previous process. Results will now be added to the clustered observation and rewards will be given by the dynamic scripting base from the effectiveness of the action.

Then again iteration will be made. Similarity matching will send data to the OSS and observation and indices will be sent and it will now choose the observation with the highest reward and command the agent on what to do next. The final part of the A.I. is the part which monitors the effectiveness of the decisions made by the A.I. If the system sees that consecutive high rewards is given it will trigger the OSS to choose an observation with lower reward point next iteration.

The proponents created an A.I. embedded with combined algorithms of dynamic scripting, case-based reasoning, and Theory of Incongruity on the algorithm. Then the evaluation will be conducted with completely randomized design of experimental design methodology to measure the effectiveness of the combined algorithms of the A.I. These algorithms were incorporated in a well-known gaming platform.

\section{PROBLEM STATEMENT}

Most of the available adaptive A.I., processes its method at a speed not enough for the player to feel its essence or rather effect in the game environment. What is the effect of the implementation of Case-Based reasoning in Adaptive A.I. on the performance of the player? Does it result in a faster speed?

1) How can the developed A.I achieve balance in its difficulty level and implement efficiency in scaling the player's performance in the game?

\section{SOlUtion}

Combining dynamic scripting with case base reasoning and theory of incongruity would provide better performance for an adaptive type of A.I. Decisions made by the dynamic scripting would be processed rapidly with the help of CBR. Difficulty scaling will be handled by the Theory of Incongruity. Thus, combining the three will yield a hybrid A.I. that will provide efficient and fast adaptation. Upon entering the game the offline processes, "clustering of observation and game indexing" of the game will be initiated. This is where the default strategy of the A.I will be used but only with the time span of the $1^{\text {st }}$ game because after which it has learned to tweak its strategy in regards with the first assessment of the player. 


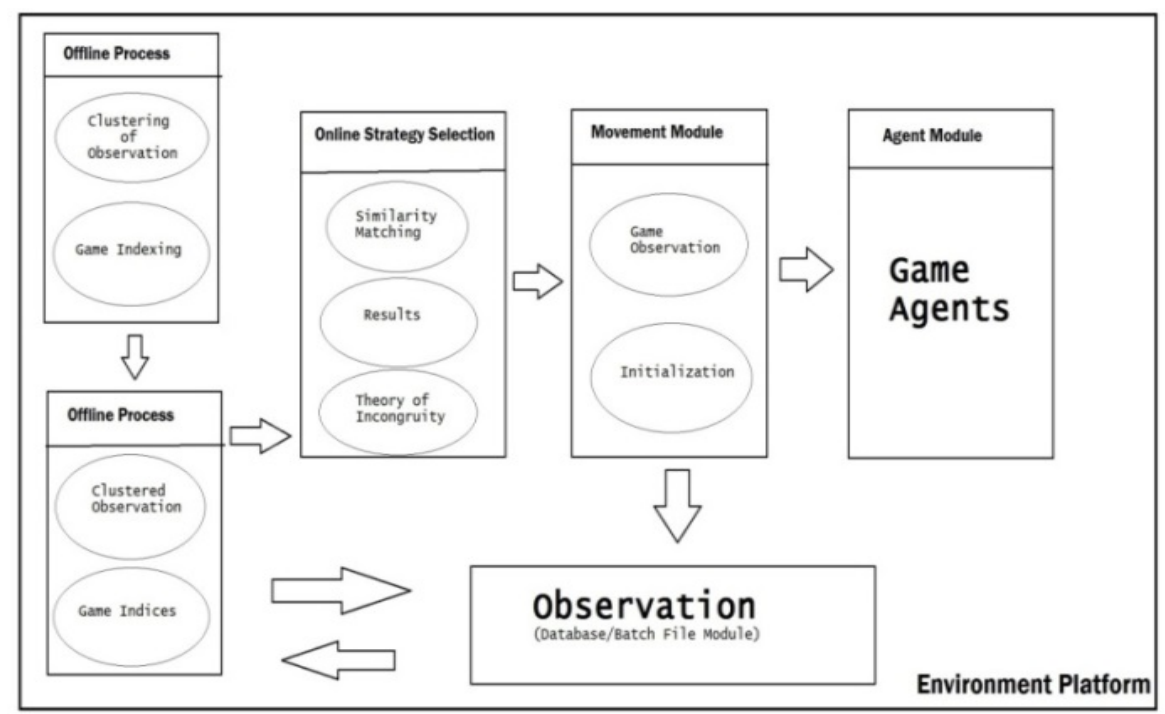

Fig. 1. System architecture

Also, the previous game observation will be loaded however; it will not be necessarily used only when a similar situation occurs during the game proper. Also the database will be initiated and ready for gathering observation about game strategies and styles that the player will use. The offline process serves as the very foundation and beginning of the adaptation of the A.I.

The next phase of the architecture would be the online strategy selection. In this phase, three processes are involved, similarity matching, analyzing of results and the injection of the concept Theory of Incongruity. As the game proper runs, this phase marks the beginning of the A.I learning through observations and the analysis of scaling what strategy to use is done but only takes effect during the next game. The similarity matching happens when the next game the strategy used changes, this means that in the A.I has a better strategy to use against the current player. This gives the A.I the edge against every player going against it, giving it a better possibility of winning.

The next process is called the process of scaling. It is a big factor knowing and monitoring game results to achieve scaling within the A.I. This brings us to the next process where inequality meets its road to balance. As every game ends and during the game proper scores and game results are taken into consideration as far as scaling is concerned. It serves as the basis in adapting the concept of Theory of Incongruity into the platform and A.I's performance in such way the games statistics would not be always the A.I winning.

The movement module functions as the eyes and data gathering module for the A.I, it is also serves as the bridge for the A.I to see and affect the game agents. The module comprises of two processes namely, the game observation and the initialization. The game observation is the eye or eyes that gather data among the platform and game agents and create a script of text file. If such codes or strategy have not been seen nor stored within the data observation (pool of strategies) that can be later used in another set of games and players prior to the offline process discussed earlier.

Meanwhile, initialization is similar to the human body's nervous system that signals the game agents of the A.I to move. Lastly, the observation module, in this module stored are the text files and scripts the A.I learns and the developers hardcoded default levelling strategies that can be accessed anytime starting from the very beginning of the system architecture.

\section{ANALYSIS}

Evaluation and data gathering were done through the method of conducting a survey among a certain sample of the population of gamers familiar with the platform used for implementation which is StarCraft. Every player is categorized according to their specific skill level. One factor why surveying was used was to allow the observation of the reaction of the player during and after the game play. This is very important in implementing the Theory of Incongruity and as well as a very good determination of success in the performance of the adaptive A.I. It is expected that players will have a constant, if not gradually increasing tension with the A.I, the players become more competitive as the game goes deeper.

TABLE I: LIKERT SCALING FOR THE ADAPTIVE

\begin{tabular}{cc}
\hline Rating & Verbal Response \\
\hline 1 & Strongly Disagree \\
2 & Disagree \\
3 & Undecided \\
4 & Agree \\
5 & Strong Agree \\
\hline
\end{tabular}

The problem with this method is how to measure reaction of one's self. Prior to the problem, the study made use of a survey designed with a scaling system in order to quantify the unmeasureable aspect needed for the study and Mathematics maybe applied. In this way, it is determined if the study is a success and user requirements and feedback were gathered and used for further improvement in any module of the study and system. In relation with the survey, the Likert Scale was used to quantify the emotions, reactions and experiences the players achieved. With the Likert Scale, it is part of the 
concept to create the set of potential scale items, it could either be rated one (1) - five (5) or one (1) - seven (7) disagree - agree response scale. Regarding with the study, the one (1) - five (5) rating scale was used. (See Table I)

The mean per question was computed to know the average results, using basic statistical computations and there on compared with the results with the other levels. The formula used is as follows:

$$
\text { Mean }=\frac{(X 1+X 2+X 3+\cdots \ldots+X n)}{N}
$$

where $N=$ the total number of variables.

The computed mean was used with the the final comparison method which is the chi-square statistic method that was applied to conduct a comparison between the default A.I and the adaptive A.I.

\section{Satisfactorylevel $=$ (no. ofpositivefeedback / numberofgamesconducted) $\times 100$}

This formula was used in comparison of the default A.I and adaptive A.I using the raw data presented or rather gathered through the survey forms.

\section{SIMULATION AND EXPERIMENTATION}

A comparison between the default A.I developed by the game makers officially and the group's developed adaptive A.I is needed especially form the external players for the purpose of confirmation of a better game experience and a confirmation that the algorithm used and injected in the game platform is a success. Twenty-Five players are asked to play divided equally into three levels as enumerated: Beginner, Intermediate, and Professional.

This is for the purpose of data segregation and proper management of the data gathering. With such strategy, it is possible for the developer and user to monitor if the adaptive A.I is functioning as it should especially the scaling feature of it.

In this way, the possibility of levelling per game skill will be emphasized and appropriate data will be clustered and grouped accordingly. After each game, a survey is answered to give a concrete measurement of the gaming experience the developers want to achieved, evaluation is done prior to a person's expression is not tangibly possible to measure with any unit of measurement, but with this study the factor of the emotions a player/users endures is counted.

TABLE II: SATISFACTORY LEVEL OF THE A.I'S RESPONSIVENESS

\begin{tabular}{llll}
\hline Beginner & $\begin{array}{l}\text { Inter } \\
\text {-mediate }\end{array}$ & $\begin{array}{l}\text { Profess } \\
\text { ional }\end{array}$ & Satisfactor \\
& & y Level
\end{tabular}

\begin{tabular}{lllll}
\hline Default & 6 & 2 & 1 & $37.50 \%$ \\
Adaptive & 8 & 5 & 3 & $66.67 \%$ \\
\hline
\end{tabular}

Table III is a comparison in regards with the overall responsiveness and performance responsiveness of the default A.I and the Adaptive A.I where CBR was implemented and surprisingly it was computed that with the concept of case-based reasoning implemented the responsiveness of the A.I is up by $29.17 \%$ from the default A.I percentage. In this way we can quantify a measurement of the A.I performance and justify that case-based reasoning is an effective way of making the A.I faster in more than $50 \%$ of the original performance.

TABLE III: OVERALL SATISFACTORY RATE OF THE ADAPTIVE A.I

\begin{tabular}{ccccc}
\hline & Beg. & Int. & Prof. & SL. \\
\hline Default & 6 & 0 & 0 & $25 \%$ \\
Adaptive & 8 & 6 & 3 & $70.83 \%$ \\
\hline
\end{tabular}

Table III depicts that the study was a success giving the adaptive A.I an edge over the default A.I by more than twice the percentage got by the default A.I. seen on the Satisfactory Level (SL) computed. Therefore it is safe to say that the answer for the statement of the problem is by implementing a one case-based reasoning algorithm as an initialization method for the A.I to perform at faster rate deliberating decision and making moves at a real time basis, and the theory of congruity for the scaling purposes.

TABLE IV: SUMMARY OF OVERALL RESULTS

\begin{tabular}{|c|c|c|c|c|}
\hline Questions & \multicolumn{2}{|c|}{ Average Rating } & \multicolumn{2}{|c|}{$\begin{array}{c}\text { Verbal } \\
\text { Response }\end{array}$} \\
\hline Overall & Default & Adaptive & $\begin{array}{c}\text { Default } \\
\text { Somewhat }\end{array}$ & Adaptive \\
\hline $\begin{array}{r}\text { Performance } \\
(\mathrm{Q} 1-\mathrm{Q} 4)\end{array}$ & 2.97 & 3.15 & Disagree & Undecided \\
\hline $\begin{array}{r}\text { Overall Gaming } \\
\text { Experience } \\
(\mathrm{Q} 5-\mathrm{Q} 11)\end{array}$ & 2.88 & 3.19 & $\begin{array}{l}\text { Somewhat } \\
\text { Disagree }\end{array}$ & Undecided \\
\hline
\end{tabular}

As Table IV is presented a better view of comparison is made and with the use of the mean or averaged answers of the testers categorized in two sets so that factors of improvement are emphasized and as well as the progress and enhancement done to the default A.I. Q1-Q4, talks about the response and performance of the A.I. It is seen that in players of different skill levels the response of the A.I. depends on the performance to move better. If you are slow enough of moving your agents so as the A.I. Q5-Q11, talks about the gaming experience the A.I gives. At this point there are no questions asked that the adaptive A.I performed well on that aspect of comparison where it got a high rating and big difference from the default A.I. given by the testers which is up by $10.76 \%$

\section{CONCLUSION}

The Theory of Incongruity was a success because the A.I. was able to create a scale in order to adjust its performances based from the level of the skill of the player. The results yielded from chapter 4 specifically questions number 11 and 
24 give the result of $65.57 \%$ versus $20 \%$ of agreement in favour of the adaptive A.I. After every game the A.I. will try to scale up or down dependent on the observed skill of the player. Thus the next time the player plays, the A.I can adjust and try to pull him up and play better. With the implementation of dynamic scripting it was possible to create an efficient and dependable scale that aids the A.I. in its decision making thus, controlling its agent in the most efficient and advantageous ways.

Here are some enhancements that can be made:

1) Enhance the algorithm so that it can mimic or copy the exact moves and decisions the player used;

2) Design the algorithm to be able to handle more unique and versatile squad groups that can handle more complex strategies; and

3) Make a way to be able to penetrate the API so that it won't be necessary to use an outside interface to get specific data of the human user.

\section{ACKNOWLEDGMENT}

The researchers would like to extend their heartfelt gratitude and thanks to the following individuals who had contributed to the success of this study and research: First of all to our God Almighty, who has bestowed each and every one of us wisdom and guidance to stay on the right path at all times even at the darkest hours of our lives; to our dear parents, for their limitless support to all our endeavours and the love given to the researchers; and lastly, to the Computer Science faculty of the ICS department of the Faculty of Engineering, University of Santo Tomas for giving the opportunity and guidance in accomplishing the goals of the study.

\section{REFERENCES}

[1] A. T. Abraham and K. McGee, "AI for dynamic team-mate adaptation in games," in Proc. of 2010 IEEE Symposium (CIG), pp. 419-426, 2010.

[2] J. R. Ludwig. (December 2008). Extending dynamic scripting., Illinois, Michigan. United States. [Online]. Available: http://hdl.handle.net/1794/9222

[3] O. Missura and T. Gartner, "Player Modeling for Intelligent Difficulty Adjustment," in Proceedings of the 12th International Conference on Discovery Science, pp. 197-211, 2010.

[4] G. V. Lankveld, P. Spronck, H. J. V. D. Herik, "Incongruity-Based adaptive game balancing. Netherlands," in Proc. of ACG'09 Proceedings of the 12th international conference on Advances in Computer Games, pp. 208-220, 2008.

[5] S. Bakkes, P. Spronck, and H. J. V. D. Herik, "Opponent modelling forcase-based adaptive game AI. Tilburg, Netherlands," Entertainment Computing, vol. 1, no. 1, pp. 27-37, 2011.

[6] S. Ontanon, K. Mishra, N. Sugandh and A. Ram, "Case-based planning and execution for real time strategy games. Atlanta," in Proceedings of the 7th international conference on Case-Based Reasoning: Case-Based Reasoning Research and Development, pp. 164-178, 2007.

[7] S. Bakkes, P. Spronck, and J. V. D. Herik, "Rapid adaptation of video game AI," 2008 IEEE Symposium (CIG), pp. 79-86, 2011.

[8] M. Fagan and P. Cunningham, "Case-based plan recognition in computergames," in Proc. of the 5th international conference on Case-based reasoning: Research and Development, Dublin, Irelan, pp. 161-170, 2011
[9] Dasgupta, Dynamic programming, algorithms, 2007.

[10] M. V. D. Heijden, S. Bakkes, and P. Spronck, "Dynamic formations in real time strategy Games," in Proc. of 2008 IEEE Symposium (CIG), pp. 47-54, 2008

[11] T. Timuri, P. Spronck, J. Van D. Herik, "Automatic rule ordering for dynamic scripting," in Proceedings of the Third Artificial Intelligence and Interactive Digital Entertainment Conference, pp. 49-54, 2007.

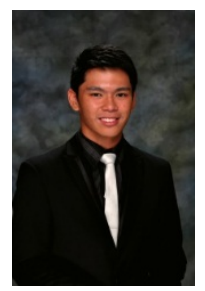

Kevin N. Francisco was born in Las Piñas City, Philippines at the Perpetual Help Hospital on May 7, 1992. He has been a consistent outstanding student from grade school to college, graduating from High School with Honorable Mention and Leadership and service award. Currently, he is studying at the University of Santo Tomas working towards a degree in Computer Science, where he is in the Dean's List. He is also active in various student organizations including the Compute Science Society. He completed his internship at Accenture as a tester of software and assistant programmer.

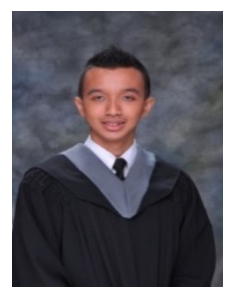

Johann-Paulo L. Ruste was born in Manila Philippines on November 20, 1992. He is currently taking up Bachelor of Science major in Computer Science at the University of Santo Tomas.

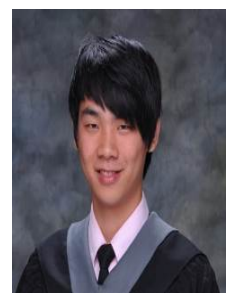

Yun Seop Song was born in Incheon, Korea on May 13, 1991. He is currently taking up Bachelor of Science major in Computer Science at the University of the Santo Tomas.

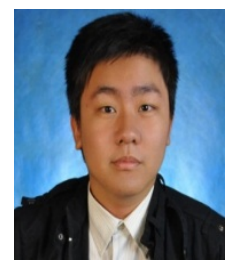

Darl Sander Sy G. was born in Manila, Philippines on September 10, 1992. He is currently taking Bachelor of Science major in Computer Science at the University of Santo Tomas and now on his 4th year. $\mathrm{He}$ was a web developer during his On the Job Training at QuestronixCorpotation, and developed software for Tae Sung Philippines, at Epza, Cavite. $\mathrm{He}$ is an active member of the Computer Science Society in UST and a former member of UST-TAG.

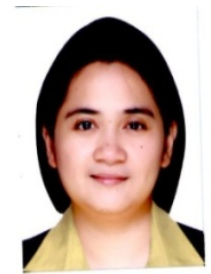

Ria A. Sagum was born in Laguna, Philippines on August 31, 1969. She took up Bachelor in Computer Data Processing Management from the Polytechnic University of the Philippines and Professional Education courses at the EulogioAmang Rodriguez Institute of Science and Technology. She received her Master of Computer Science degree from the De La Salle University in 2012.

She is currently teaching at the Department of Computer Science, College of Computer Management and Information Technology, Polytechnic University of the Philippines in Sta. Mesa, Manila and a lecturer at the Information and Computer Studies, Faculty of Engineering, University of Santo Tomas in Manila.

Ms. Sagum has been a presenter in different conferences, including the 2012 International Conference on e-Commerce, e-Administration, e-Society, e-Education, and e-Technology and National Natural Language Processing Research Symposium. She is a member of different professional associations including ACMCSTA and an active member of the Computing Society of the Philippines- Natural Language Processing Special Interest Group. 